\title{
Sex Risk among Young Men who have Sex with Men who use Grindr, a Smartphone Geosocial Networking Application
}

Eric Rice ${ }^{1 *}$, lan Holloway ${ }^{2}$, Hailey Winetrobe ${ }^{1}$, Harmony Rhoades' ${ }^{1}$, Anamika Barman-Adhikari ${ }^{1}$, Jeremy Gibbs ${ }^{1}$, Adam Carranza $^{1}$, David Dent $^{1}$ and Shannon Dunlap ${ }^{1}$

${ }^{1}$ School of Social Work at the University of Southern California, USA

${ }^{2}$ Department of Social Welfare, Luskin School of Public Affairs, University of California Los Angeles, USA

\begin{abstract}
Objectives: Smartphone geosocial networking applications, like Grindr, have become a new context through which young men who have sex with men (YMSM) can meet potential sex partners. Geosocial networking applications move beyond online social networking websites like Facebook by utilizing smartphones' geo-location functions to facilitate connections with other users based on their current physical location. This study presents data on HIV risk-taking behaviors of YMSM who use Grindr, comparing the sex behaviors with partners met via the application to behaviors with partners met via other means (e.g., a bar, through friends, online).

Methods: Utilizing the geo-locating feature of Grindr, 195 YMSM, aged 18 to 24, were randomly recruited based on their location within West Hollywood and Long Beach, CA between August and October, 2011. Participants completed an online survey.

Results: YMSM reported using Grindr for entertainment, socializing, partner seeking, and gay community connection. Seventy-five percent of users reported sexual encounters with partners met on Grindr. YMSM reported significantly higher rates of condom use with partners met on Grindr (59.8\%) relative to those partners met elsewhere $(41.9 \%)$. Only $14.7 \%$ reported unprotected anal intercourse (UAI) with their last Grindr partner. YMSM who reported UAI with their last partners from Grindr reported significantly more recent male anal sex partners than YMSM who reported no UAI with their last Grindr-met partners.

Conclusions: Grindr was used by YMSM for a variety of reasons, and not exclusively for the purpose of sex partner seeking. Overall YMSM who use Grindr practice safer sex with partners met via the application than with partners met elsewhere. YMSM engaging in UAl with partners on Grindr are an especially high-risk group and should be targeted by prevention efforts. Sexual risk behavior with geosocial networking-located partners could be addressed with mobile HIV prevention applications, or within Grindr and other similar applications.
\end{abstract}

Keywords: Young men who have sex with men (YMSM); Grindr; Sexual risk behavior; Geosocial networking applications; HIV prevention

Abbreviations: YMSM: Young Men Who Have Sex With Men; MSM: Men Who Have Sex With Men; UAI: Unprotected Anal Intercourse; App: Application

\section{Introduction}

Young men who have sex with men (YMSM) remain at high risk for HIV infection and continue to account for more than one-quarter of all new infections in the United States, and over two-thirds amongst 13-29 year olds [1]. In today's digital world, it is becoming increasingly common for MSM of any age to find sex partners through the internet [2-7], and more recently through smartphone geosocial networking applications, such as Grindr. Popular media outlets have expressed concern that geosocial networking applications promoting dating and sex partner-locating are a facilitator of sexual risk behaviors [8,9]. The goal of this study was to utilize Grindr's geo-locating features to generate a random sample of YMSM Grindr users and assess the sexual risk behaviors associated with this application by comparing their most recent sexual encounter, whether meeting the partner through Grindr or elsewhere.

\section{Grindr: a geosocial networking application}

Geosocial networking applications move beyond the connectivity of online peer-to-peer social networking websites, such as Facebook or Twitter. This new form of social networking employs smartphones' geo- locating capabilities to facilitate connections with other users based on their current physical location. These technologies have created a faster and easier way for YMSM to meet potential partners based on shared interests, attraction, and physical proximity. Grindr is currently a popular geosocial networking application, which targets men who have sex with men (MSM). The application boasts more than 3 million users in 192 countries [10], and with an estimated 8,000 users joining the Grindr community every day [11], the application has proven to be a phenomenon-connecting its users discreetly, conveniently, and all for free (or a low monthly fee for its premium features).

The basic Grindr interface is five rows of four thumbnail size profile pictures, appearing sequentially based on geographic proximity, with the closest users appearing first. Men who want to communicate with another user touch the thumbnail picture to be directed to a full screen profile picture and information about distance (e.g., 643 feet, 2 miles),

*Corresponding author: Eric Rice, Ph.D, School of Social Work at the University of Southern California, USA, Tel: 213-743-4766; Fax: 213-743-2341; E-mail: ericr@usc.edu

Received June 04, 2012; Accepted July 10, 2012; Published July 12, 2012

Citation: Rice E, Holloway I, Winetrobe H, Rhoades H, Barman-Adhikari A, et al. (2012) Sex Risk among Young Men who have Sex with Men who use Grindr, a Smartphone Geosocial Networking Application. J AIDS Clinic Res S4:005. doi:10.4172/2155-6113. S4-005

Copyright: (c) 2012 Rice E, et al. This is an open-access article distributed under the terms of the Creative Commons Attribution License, which permits unrestricted use, distribution, and reproduction in any medium, provided the original author and source are credited. 
Citation: Rice E, Holloway I, Winetrobe H, Rhoades H, Barman-Adhikari A, et al. (2012) Sex Risk among Young Men who have Sex with Men who use Grindr, a Smartphone Geosocial Networking Application. J AIDS Clinic Res S4:005. doi:10.4172/2155-6113.S4-005

Page 2 of 8

age, when the user was last online, and any additional profile information the user posts about himself. On this screen, there is an option that allows users to exchange text messages within the application and text others their exact physical location on a map.

\section{Possible motivations for grindr use}

Although the popular press presents Grindr as a "gay hook-up app," there is no information about the sexual risk-taking behaviors of YMSM who use Grindr or other geosocial networking applications. The most relevant extant work pertains to YMSM seeking sex partners on the internet and associated sex risk behavior; yet this body of research has many inconsistent findings. While a 2006 meta-analysis found that MSM seeking sex partners on the internet were more likely to have unprotected anal intercourse (UAI) than their counterparts who found their partners offline [5], more recent research in this area does not find behaviors with internet-met sex partners to be riskier than behaviors with offline-met sex partners amongst YMSM who seek partners both online and offline $[7,12-14]$. In fact, some studies indicate that sex with partners met online is less risky, due to increased odds of disclosing HIV and sexually transmitted infection status to partners met online [12], and a reduced likelihood of engaging in sex under the influence of drugs or alcohol with those partners [12,13].

Despite these findings of reduced sexual risk behavior when comparing online- and offline-met sexual partners, studies have found higher overall numbers of sex partners among MSM who meet partners online [15,16]. Rosser et al. [16] found that MSM reported more UAI with online-met partners than with offline-met partners, but because of the higher number of partners met online, it appeared that MSM with online-met partners had significantly more UAI overall. Additionally, among YMSM who recently engaged in sex with an internet-met partner and used an online dating website, Bauermeister [17] found that time spent looking online for casual sex partners was positively correlated with the number of recent UAI partners. As such, some have concluded that the internet simply may be another means for MSM to meet partners and is not causally related to condom-using behaviors $[13,15,16]$.

\section{Sampling using geosocial networking apps}

YMSM may be using geosocial networking applications for a variety of reasons. The geo-locating aspect of the application may make it easier to connect with other YMSM, even as compared to MSMoriented online communities (e.g., gay.com, Manhunt, Adam4Adam). Again, one must consider the existing literature on online partner seeking when examining the psychosocial motivations for using these applications. Evidence suggests that YMSM engage in online partner seeking to avoid stigma [18,19], as an easier means of approaching men [20], for its safety and its convenience [21], and because it affords men the opportunity to manage and reveal their sexual identity in a personalized way [22,23]. Grindr's founder Joel Simkhai believes the application to also be a tool in helping YMSM to make new friends, begin new relationships, and to assist in the coming out process [24].

Grindr and other geosocial networking applications provide researchers with an innovative tool for sampling and recruiting YMSM into HIV/AIDS epidemiological and prevention studies. Such applications provide an immediate, virtual sampling frame where demographic or location screening is unnecessary. The interfaces for these applications typically rank users according to geographic proximity to the user. Using a random number generator, researchers can easily randomly select users as they appear in the interface.
Moreover, user profiles of such applications typically include basic demographic information such as age and race, which can be used to automate some aspects of eligibility assessments, reducing the potential participant burden associated with screening.

There are advantages of using these applications as a sampling tool. First, these applications allow for the random sampling of MSM within the areas where sex is being sought, without being limited to specific venues such as gay bars, clubs, or pride festivals. As with traditional venue-based sampling, when recruiting in gay neighborhoods, MSM who live in other neighborhoods, but who travel to these neighborhoods for work or socialization are easily included in the sampling frame. Moreover, researchers can sample men who live in areas outside of traditional gay neighborhoods, including hidden MSM populations or men who are on the "down low," that is MSM who identify as heterosexual. Second, a small research team can reach a large number of potential participants quickly by sending pre-written text messages. Third, recruitment can be done at any time of day, given that many users are logged into the application throughout the day, unlike many venue-based approaches that rely on recruitment at night when potential respondents may be unable to participate due to alcohol and substance use, especially at bars and clubs. With increasing numbers of YMSM utilizing this technology each year [25], smartphone geosocial applications may provide a valuable and cost-effective modality for sample recruitment. Additionally, a recent panel of experts in the field of at-risk MSM indicated the need for further investigation of novel sampling techniques that enhance what is known about this population [26].

The aims of the current study were to assess YMSM motivations for using smartphone geosocial networking applications and users' potential HIV risk-taking behaviors associated with meeting persons from the application. The study utilized the geo-locating features of the application to collect a random sample of Grindr users from West Hollywood and Long Beach, CA. The study assessed: (1) the primary uses of the application; (2) the number of YMSM who used the application to find sex partners; and (3) whether individuals whose last sexual encounter was with a Grindr-met partner engaged in riskier sex behavior than those whose last partner was found by other means (e.g., at a bar, introduced by friends, on the internet). Because the sample was collected only among Grindr users, assessing whether Grindr users have riskier sexual behaviors than non-Grindr users is beyond the scope of the current data.

\section{Methods}

\section{Recruitment}

Recruitment occurred in two Los Angeles County cities with large populations of YMSM: Long Beach and West Hollywood. Research staff (recruiters) created Grindr profiles that identified themselves as researchers. Recruiters positioned themselves centrally within the target city, launched the Grindr application, and randomly selected 18-24 year old Grindr users within a five to seven mile range. Grindr provides a screen with five rows of four profiles per row. Recruiters had a random number generator to select one potential participant randomly from each row. After the fifth potential participant was contacted, the recruiter moved forward in the application interface to the next five rows of potential participants and continued this procedure. The application automatically records all text messages sent and received. Using this feature, recruiters were able to ensure that no user was approached more than once. If a user was randomly selected a second time, the recruiter moved on to the next row of users and 


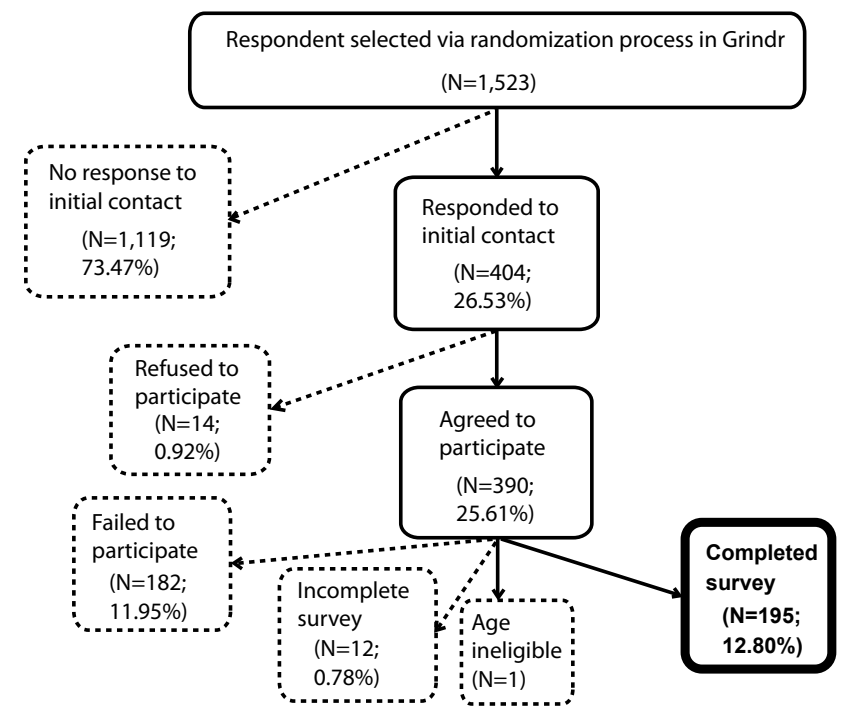

Figure 1: Participation Rates among Grindr-Recruited YMSM, Los Angeles CA, 2011.

continued the random selection procedure outlined above. Recruiters documented the distance between the recruiter and each selected user at the time of recruitment.

Those users who were randomly selected to participate in the study were sent an initial message containing information about the study, a unique login code, and a link to complete the anonymous online survey. If users responded, the recruiter exchanged text messages with the potential participant to answer any questions about the study. After entering the unique login code, participants had the opportunity to read an informed consent page, which included the investigator's contact information for any questions. Participants who completed the survey received a $\$ 25$ downloadable gift card. All procedures were approved by the Institutional Review Board at the University of Southern California.

Between August 8, 2011 and October 3, 2011 the two recruiters approached 1,523 YMSM. Details about recruitment are provided in Figure 1. Of the 1,523 men approached via the application, $26.5 \%$ responded to the recruiters and $25.6 \%$ agreed to participate. Of those men who agreed to participate (390), 50.0\% (195) completed the survey. Overall, $12.8 \%$ of the men approached via Grindr text message completed the survey. Only eligible participants were randomly selected, so our overall response rate is calculated based on the proportion of those contacted who completed the survey, without any additional inflations of this rate based on excluding non-eligible participants from our calculations. The one exception is identified in the figure, in which one participant was outside of the age range. His profile likely listed his age as between 18 and 24 years old, however, in the survey he reported his age as 25 years old, and thus was promptly deemed ineligible for the study.

\section{Measures}

Demographic variables are presented in Table 1. Participants were asked their age in years, race/ethnicity, sexual orientation, highest level of education completed, employment status, and relationship status. Race/ethnicity data was collected to determine any potential associations with increased risk behavior since racial/ethnic minority YMSM are at greatest risk of new HIV infections [1]. Respondents selfreported their race/ethnicity with the following question and response choices: "What is your race? (Select one response): (1) Black or African American, (2) Hispanic/Latino, (3) White, (4) Asian, (5) Native Hawaiian or Other Pacific Islander, (6) American Indian or Alaska Native, (7) Mixed race, (8) Other." Participants had the opportunity to complete an open textbox for "mixed race" or "other." Sexual orientation response options were: "(1) Homosexual (gay or lesbian), (2) Bisexual, (3) Heterosexual (straight), (4) Questioning/Unsure, (5) Queer, (6) Other;" an open text box was available for "other." Participants were also asked to describe their sexual attraction to both males and females on a four-point scale from "not at all attracted" to "very strongly." Education completion ranged from less than high school to a doctorate degree. Participants were asked about their current relationship status; non-single participants further described their relationship as either with a life partner or boyfriend/girlfriend, and as either monogamous or open.

A series of questions assessed Grindr use patterns of participants: how frequently they logged on to the application, the length of time they had been a Grindr user, the content of their profile photo(s), the

\begin{tabular}{|c|c|}
\hline Variable & N (\%) \\
\hline Mean age (range 18-24) (SD) & $21.8(1.7)$ \\
\hline \multicolumn{2}{|l|}{ Race } \\
\hline Black & $9(4.6)$ \\
\hline Latino/Hispanic & $64(32.8)$ \\
\hline White & $76(39.0)$ \\
\hline Asian/Pacific Islander & $19(9.7)$ \\
\hline Mixed & $24(12.3)$ \\
\hline Other & $3(1.5)$ \\
\hline \multicolumn{2}{|l|}{ Sexuality } \\
\hline Homosexual & $168(86.6)$ \\
\hline Bisexual & $18(9.3)$ \\
\hline Heterosexual & $1(0.5)$ \\
\hline Questioning & $4(2.1)$ \\
\hline Queer & $1(0.5)$ \\
\hline Other & $2(1.0)$ \\
\hline \multicolumn{2}{|l|}{ Attraction to men } \\
\hline Very strongly & $168(86.2)$ \\
\hline Somewhat strongly & $23(11.8)$ \\
\hline Not very strongly & $4(2.1)$ \\
\hline \multicolumn{2}{|l|}{ Attraction to women } \\
\hline Very strongly & $3(1.5)$ \\
\hline Somewhat strongly & $25(12.8)$ \\
\hline Not very strongly & $63(32.3)$ \\
\hline Not at all & $104(53.3)$ \\
\hline \multicolumn{2}{|l|}{ Education } \\
\hline Less than high school & $5(2.6)$ \\
\hline High school or GED & $25(12.8)$ \\
\hline Some college & $95(48.7)$ \\
\hline Bachelors degree & $63(32.3)$ \\
\hline Masters or professional degree & $7(3.6)$ \\
\hline Employed & $129(66.2)$ \\
\hline \multicolumn{2}{|l|}{ Relationship status } \\
\hline Single & $170(87.2)$ \\
\hline Male spouse - monogamous & $2(1.0)$ \\
\hline Male spouse - open & $1(0.5)$ \\
\hline Female spouse - monogamous & $1(0.5)$ \\
\hline Boyfriend - monogamous & $16(8.2)$ \\
\hline Boyfriend - open & $5(2.6)$ \\
\hline
\end{tabular}

Table 1: Demographic Characteristics of Grindr Users in West Hollywood and Long Beach, CA, $2011(\mathrm{~N}=195)$. 
Citation: Rice E, Holloway I, Winetrobe H, Rhoades H, Barman-Adhikari A, et al. (2012) Sex Risk among Young Men who have Sex with Men who use Grindr, a Smartphone Geosocial Networking Application. J AIDS Clinic Res S4:005. doi:10.4172/2155-6113.S4-005

Page 4 of 8

\begin{tabular}{|l|l|}
\hline Variable & N (\%) \\
\hline How often do you log on to Grindr? & $97(49.7)$ \\
\hline 5 or more times a day & $56(28.7)$ \\
\hline More than once a day but less than 5 times per day & $19(9.7)$ \\
\hline Once a day & $16(8.2)$ \\
\hline A few days a week & $4(2.1)$ \\
\hline About once a week & $3(1.5)$ \\
\hline Less than once a week & \\
\hline When did you start using Grindr? & $25(12.8)$ \\
\hline Less than 1 month ago & $57(29.2)$ \\
\hline More than 1 month, but less than 6 months ago & $37(19.0)$ \\
\hline More than 6 months, but less than 1 year ago & $76(39.0)$ \\
\hline More than 1 year ago & $161(82.6)$ \\
\hline Is your face visible on your Grindr profile? (Yes) & \\
\hline In your photo do you show any of the following naked body parts? & $54(27.7)$ \\
\hline Chest & $39(20.0)$ \\
\hline Abs & $54(27.7)$ \\
\hline Arms/Biceps & $15(7.7)$ \\
\hline Back/Shoulders & $2(1.0)$ \\
\hline Legs & $121(62.1)$ \\
\hline None & \\
\hline When you use Grindr, what time of day is it usually? & $105(53.8)$ \\
\hline Morning & $145(74.4)$ \\
\hline Afternoon & $141(72.3)$ \\
\hline Evening & $143(73.3)$ \\
\hline Night (before midnight) & $103(52.8)$ \\
\hline Late night (after midnight) & $4(2.1)$ \\
\hline Do you primarily use Grindr on: & $15(7.7)$ \\
\hline Weekends & $175(90.2)$ \\
\hline Weekdays & \\
\hline Both & \\
\hline The & \\
\hline
\end{tabular}

The last time you used Grindr, was it during or immediately after you had been drinking or using drugs?

\begin{tabular}{|l|l|}
\hline Yes & $16(8.2)$ \\
\hline No & $170(87.2)$ \\
\hline Don't know & $9(4.6)$ \\
\hline What are the reasons you use Grindr? & \\
\hline To make new friends & $155(79.5)$ \\
\hline To meet people to have sex/hook up with & $127(65.1)$ \\
\hline To find someone to date & $126(64.6)$ \\
\hline To "kill" time & $166(85.1)$ \\
\hline To connect to the gay community & $127(65.1)$ \\
\hline To find people to drink/use drugs with & $23(11.8)$ \\
\hline To communicate with in-person friends & $60(30.8)$ \\
\hline To reconnect with people from the past & $24(12.3)$ \\
\hline What is your number one reason for using Grindr? & \\
\hline To make new friends & $40(20.5)$ \\
\hline To meet people for to have sex/hook up with & $52(26.7)$ \\
\hline To find someone to date & $43(22.1)$ \\
\hline To "kill" time & $42(21.5)$ \\
\hline To connect to the gay community & $14(7.2)$ \\
\hline Other & $4(2.1)$ \\
\hline Ever had sex with a partner met on Grindr & \\
\hline Yes & $147(75.4)$ \\
\hline No & $48(24.6)$ \\
\hline Mean number of lifetime male anal sex partners (SD) & $31.8(87.0)$ \\
\hline Mean number of recent (past 30 day) male anal sex partners (SD) & $2.5(7.7)$ \\
\hline
\end{tabular}

Table 2: Grindr Use Characteristics and Behaviors ( $\mathrm{N}=195)$. time and day of typical use, concurrent substance and Grindr use, and motivations for using Grindr. The exact wording of these items appears in Table 2.

Finally, participants were asked a series of questions regarding their last sexual encounter for both partners met on Grindr and partners not met on Grindr. These included: type of partner (i.e., life partner, boyfriend/girlfriend, or casual sex partner), occurrence of last sexual encounter, engagement in sex under the influence of drugs or alcohol, and engagement in specific sexual activities (i.e., UAI, anal sex with a condom, unprotected vaginal sex, vaginal sex with a condom, oral sex without a condom, oral sex with a condom, or no penetration or oral sex). The sexual activity variables are not mutually exclusive categories; participants were encouraged to "check all that apply." Count variables were used to assess number of lifetime and past 30-day vaginal and anal (female and male) sex partners, and number of past 30-day sex partners met on Grindr.

\section{Data analysis}

Bivariate analyses were conducted with the sub-sample of YMSM who reported data on sexual encounters with persons met through Grindr $(\mathrm{N}=144)$ to assess differences between sex behaviors, including UAI, based on where YMSM met their most recent sex partner. Three respondents were dropped from these analyses either because they reported their most recent sex partner as female or did not report specific sexual activity with their most recent Grindr-located sex partner. Associations were established using independent sample $t$-tests for continuous variables and Chi-square or Fisher's Exact tests for categorical variables. Frequencies reported in these analyses can be assumed to have a $95 \%$ confidence interval within plus or minus $7 \%$ of a given parameter estimate, as we have 195 observations taken from a population of over 3 million. This is based on a parameter of $50 \%$. The size of the $95 \%$ confidence interval shrinks to plus or minus 1.4 as estimates approach either $1 \%$ or $99 \%$. As $7 \%$ is the maximum 95\% confidence interval, this allows for a conservative estimate for the parameters. All analyses were conducted using SPSS, Version 19 (SPSS, Inc., Chicago, IL).

\section{Results}

Respondents were predominantly White and Latino, had attended at least some college, and identified as homosexual (Table 1). Most participants reported strong sexual attraction to men, and no sexual attraction to women. Eighty-seven percent of the sample reported being single, with only $8.2 \%$ reporting being in a monogamous relationship with a boyfriend (Table 1).

Table 2 presents data on Grindr usage patterns. Three-fourths of YMSM (75.4\%) reported ever having sex with a partner met on Grindr. On average, YMSM Grindr-users reported 31.8 lifetime male anal sex partners and 2.5 male anal sex partners in the prior 30 days. Overall, $85.1 \%$ used the application to "kill time," $79.5 \%$ used it to make new friends, $65.1 \%$ to connect to the gay community, $65.1 \%$ to meet people to have sex/hook-up with, and $64.6 \%$ to find someone to date. Approximately half of the participants (49.7\%) reported using Grindr more than five times per day, and over half $(58.0 \%)$ reported using the application for six months or longer at the time of the interview. Most men $(82.6 \%)$ reported that their face was visible in their profile picture.

Among men who have ever had sex with a Grindr-met partner, there are significant differences in sexual encounters between those who met their last partner on Grindr, and those who met their last partner elsewhere (Table 3). The majority of YMSM whose most recent 
Citation: Rice E, Holloway I, Winetrobe H, Rhoades H, Barman-Adhikari A, et al. (2012) Sex Risk among Young Men who have Sex with Men who use Grindr, a Smartphone Geosocial Networking Application. J AIDS Clinic Res S4:005. doi:10.4172/2155-6113.S4-005

Page 5 of 8

\begin{tabular}{|c|c|c|c|c|c|}
\hline & $\begin{array}{l}\text { Total } \\
(\mathrm{N}=144)\end{array}$ & $\begin{array}{l}\text { Not Grindr-Met Partner } \\
(\mathrm{N}=62)\end{array}$ & $\begin{array}{l}\text { Grindr-Met Partner } \\
(\mathrm{N}=82)\end{array}$ & & \\
\hline Variable & $\mathrm{N}(\%)$ & $N(\%)$ & $\mathrm{N}(\%)$ & $x^{2}$ ort & $p$ \\
\hline \multicolumn{6}{|l|}{ How would you describe your last sexual partner? } \\
\hline Life partner/boyfriend $^{b}$ & $25(17.5)$ & $21(33.4)$ & $4(4.9)$ & 21.17 & $<0.001$ \\
\hline Casual sex partner & $118(82.6)$ & $40(65.6)$ & $78(95.1)$ & & \\
\hline \multicolumn{6}{|l|}{ When was last sexual encounter? } \\
\hline Within the last week & $83(57.6)$ & $34(54.8)$ & $49(59.8)$ & 0.39 & 0.82 \\
\hline Within the last month & $34(23.6)$ & $16(25.8)$ & $18(22.0)$ & & \\
\hline More than 1 month ago & $27(18.8)$ & $12(19.4)$ & $15(18.3)$ & & \\
\hline $\begin{array}{l}\text { The last time you had sex were either you or your partner under the influence of } \\
\text { drugs or alcohol?c }\end{array}$ & $46(33.8)$ & $18(31.6)$ & $28(35.4)$ & 0.22 & 0.64 \\
\hline \multicolumn{6}{|l|}{ The last time you had sex, what kinds of sex did you have? } \\
\hline Anal sex, with a condom & $75(52.1)$ & $26(41.9)$ & $49(59.8)$ & 4.49 & 0.03 \\
\hline Anal sex, no condoms/bareback & $39(27.1)$ & $20(32.3)$ & $19(23.2)$ & 1.48 & 0.22 \\
\hline Oral sex, with a condom & $7(4.9)$ & $3(4.8)$ & $4(4.9)$ & 0.000 & 0.99 \\
\hline Oral sex, no condom & $109(75.7)$ & $48(77.4)$ & $61(74.4)$ & 0.18 & 0.68 \\
\hline No penetration and no oral sex $x^{d}$ & $4(2.8)$ & $0(0.0)$ & $4(4.9)$ & $\ldots$ & $\ldots$ \\
\hline Mean number of lifetime male anal sex partners (SD) & $38.5(98.8)$ & $25.5(29.4)$ & $37.7(73.2)$ & -1.37 & 0.17 \\
\hline Mean number of recent (past 30 day) male anal sex partners (SD) & $2.3(3.3)$ & $1.9(2.6)$ & $2.6(3.8)$ & -1.29 & 0.20 \\
\hline Mean number of recent (past 30 day) sex partners met on Grindr (SD) & $1.9(2.9)$ & $1.1(2.9)$ & $2.4(2.8)$ & -2.75 & 0.01 \\
\hline
\end{tabular}

a Three cases missing on whether last sex was from Grindr

b Two responses options of life partner and boyfriend were collapsed into one for analysis purposes

" Reponses listed are "yes" answers; other options were "no" and "I don't know"

a No comparison due to cell size of 0

Table 3. Comparison of Sexual Behaviors by Whether or Not Last Sex Partner was Met on Grindr among Those Who Reported Ever Having Sex with Someone Met on Grindr $(\mathrm{N}=144)^{\mathrm{a}}$

\begin{tabular}{|l|l|l|l|}
\hline & $\begin{array}{l}\text { No UAI with Grindr-Met Partner } \\
(\mathrm{N}=114)\end{array}$ & $\begin{array}{l}\text { UAl with Grindr-Met Partner } \\
(\mathrm{N}=29)\end{array}$ & Mean (SD) \\
\hline Variable & Mean (SD) & $53.2(97.9)$ & $t$ \\
\hline Number of lifetime male anal sex partners & $27.3(42.8)$ & $4.1(5.4)$ & -1.39 \\
\hline Number of recent (past 30 day) male anal sex partners & $1.9(2.4)$ & 0.17 & -2.21 \\
\hline
\end{tabular}

Table 4: Number of Recent and Lifetime Male Anal Sex Partners among YMSM Who Engaged in Unprotected Anal Intercourse (UAI) Versus Those Who Did Not with Las Grindr-Met Sex Partner, among Those Who Reported Ever Having Sex with Someone Met on Grindr (N=144).

partner was met on Grindr indicated that this partner was a casual sex partner, rather than a life partner or boyfriend; these men also had a higher average number of recent sex partners met on Grindr. A greater percentage of those who met their most recent sex partner on Grindr reported having anal sex with a condom during that sexual encounter.

To further elucidate whether sex risk varied by how YMSM met their partners, we compared reports of unprotected anal intercourse (UAI) among individual participants. Each participant was asked to report on their sex behaviors with their most recent Grindr-met partner and their most recent partner met elsewhere (e.g., through friends, at a bar, online). We compared the reports of UAI between these two encounters on an individual-level. Among those reporting ever having a Grindr-met partner $(\mathrm{N}=144), 14.7 \%(\mathrm{~N}=29)$ engaged in UAI with their most recent Grindr-met partner. Nearly three-quarters of the sample $(\mathrm{N}=104 ; 72 \%)$ reported no differences in UAI behaviors, regardless of how they met their two partners. Nearly one-fifth $(\mathrm{N}=28$; $19.4 \%$ ) of the sample reported UAI with their last non-Grindr-met partner, but reported using a condom with their last partner met via Grindr. Comparatively, only $8 \%(\mathrm{~N}=12)$ reported UAI with their last partner met on Grindr and condom use with their last partner met elsewhere.

Table 4 presents results on the number of lifetime and past 30-day male anal sex partners, among those who have ever had sex with a partner met on Grindr. YMSM who had UAI with their most recent Grindr-met partner reported having on average 2.2 more recent (i.e., past 30 day) male anal sex partners $(t=-2.21, p=0.04)$, compared to men who reported not engaging in UAI with their most recent Grindr-met partner.

\section{Discussion}

There are several important findings to emerge from this study. First, Grindr is not being used by YMSM exclusively for the purpose of locating sex partners. Almost one-quarter of the sample reported never having had sex with someone they met on Grindr. There were significant differences in reported frequency, duration, and patterns of use for these YMSM. The YMSM who identified as ever having sex with a Grindr-met partner reported a higher number of lifetime male anal sex partners relative to those who reported never having sex with someone they met on Grindr. This result is largely consistent 
Citation: Rice E, Holloway I, Winetrobe H, Rhoades H, Barman-Adhikari A, et al. (2012) Sex Risk among Young Men who have Sex with Men who use Grindr, a Smartphone Geosocial Networking Application. J AIDS Clinic Res S4:005. doi:10.4172/2155-6113.S4-005

Page 6 of 8

with existing findings regarding online partner seeking, which indicate increased numbers of lifetime partners among those who engage in sex with partners met online $[15,16]$. It is important to note that these results do not allow one to compare the sex risk behaviors of Grindrusers and non-Grindr-users, rather these results allow one to assess the differences in behaviors by partner type (i.e., Grindr-met versus other) among YMSM who use Grindr to locate sex partners.

While popular media portrays Grindr primarily as a "gay hook-up app," the participants of this study provided several other reasons for using the application. Along with finding sex partners, the majority of YMSM reported using Grindr for community building, entertainment, and socializing. These findings suggest that Grindr may be a positive environment for these YMSM with low barriers to entry and a reduced risk of encountering homophobia. Moreover, many MSM under the age of 21 who are excluded from gay clubs and bars may be particularly reliant on this application as a mode of connecting to other MSM. Further explorations into this issue are warranted.

For the majority of Grindr users who found sex partners via the application, sex with partners met on Grindr tended to be safer than sex with partners found by other means. These data enable us to make two important contrasts. First, across the sample, YMSM whose last partner was met on Grindr were more likely to have anal sex with a condom, as compared to men whose last partner was not met on Grindr. Second, when comparing individuals' last Grindr-met partner to their last nonGrindr-met partner, the majority reported no difference in unprotected anal sex between the two partners. Nineteen percent reported using condoms with a Grindr partner when they had UAI with their last non-Grindr partner, and only $8 \%$ reported UAI with their last Grindr partner when they had used a condom with their most recent nonGrindr partner. As with several recent studies of YMSM who engage in internet sex partner seeking [12-15], we found the majority of YMSM's condom using behaviors were unaffected by whether Grindr was the medium through which their partner was located.

Importantly, a small percentage of men reported UAI with partners met via Grindr. The 15\% of YMSM who reported not using a condom during their last Grindr-met sexual encounter also reported more recent male anal sex partners. This finding is akin to Rosser et al. [16] results, in which $5 \%$ of their sample reported a high number of unprotected anal sex partners within the past three months, although our data did not collect detailed information on UAI outside of the most recent Grindr-met and other-met partners. These results suggest that Grindr users who are engaging in UAI and have higher numbers of partners are at great risk for HIV/AIDS transmission and acquisition and are in need of targeted prevention services. These prevention services may be successful when delivered through the smartphone application in which these men are locating sex partners. Further research on the utility and acceptability of such prevention strategies for this high-risk group is needed.

Finally, this study utilized a novel random sampling method to recruit YMSM who are seeking sex via geosocial networking applications. There are benefits as well as costs to this method. The primary benefits are: (1) the application's interface can be easily used to generate a random sample in the field; (2) the application can be used to recruit YMSM samples quickly (e.g., two recruiters successfully made contact with 1,523 eligible YMSM and recruited 195 YMSM in less than 60 days); (3) the sampling strategy recruits YMSM in neighborhoods in which they work and socialize and does not depend on approaching men at specific venues such as bars, clubs, bookstores, or gay service organizations, which further allows for men on the "down low" to be recruited; and (4) both recruiter and participant burden was low.

The initial 1,523 contacts yielded 195 participants, a 12.8\% response rate. While this response rate appears low when compared to more traditional response rates from venue-based YMSM sampling [27-31], it is not unexpected given our methodology. We contacted potential respondents with a single text message and sent no follow-up messages. Application chat messages are easy to ignore, especially when compared to in-person recruitment, and there is no guarantee that potential respondents even opened the message. As such, our response rate is not reflective of actual participant refusals. This method is similar to other recruitment methods for digital venues, such as respondentdriven sampling or banner advertisements [32,33] where it is not possible to calculate true response rates. For this study, $96.5 \%$ of those who responded to the initial contact agreed to participate in the study; however, only $50.0 \%$ of those men actually completed the survey.

Two other limitations we encountered with this method indicate areas where future research can improve on application messagingbased recruitment methods in digital venues. First, our recruitment messages were initially reported as spam by Grindr users, even though the recruiter's profile clearly stated their presence was for research purposes. Future research needs to be conscientious of how messages are framed to avoid being interpreted as junk mail. Secondly, $46 \%$ of YMSM who agreed to take the survey during the text message communication never subsequently logged in to complete it. Men may have changed their minds, or simply forgotten to continue at a later time. It would be helpful to examine how quickly respondents proceed from the initial text message to actually logging in to take the survey, and incentivizing prompt follow-through may increase the response rate (i.e., $\$ 20$ for taking the survey, and an extra $\$ 5$ for taking it in the next 30 minutes).

Finally, we did not use corrections for multiplicity when examining multiple "outcomes" in this study. Indeed, there is a danger in committing Type 1 error when conducting numerous tests on multiple outcomes in a study. It should be noted that we have only conducted 12 statistical comparisons on two outcomes (10 in Table 3 and 2 in Table 4). We decided not to adjust for multiple comparisons given the exploratory nature of this study. Our power is already limited due to the small sample size. Given the fact that this is the first study to report on the association between sexual risk behavior and Grindr use, we believe that all associations should be reported with the acknowledgement that it is possible that a small percentage may be due to chance alone. Future studies with larger numbers of comparisons and larger sample sizes may want to consider using statistical procedures that adjust for multiple comparisons.

Benefits of utilizing application messaging recruitment on Grindr, as compared to venue-based sampling, include the ability to recruit YMSM under 21, who cannot legally patronize bars and $21+$ clubs where venue-based sampling often happens. This methodology may also be an important tool for recruiting hidden populations, such as men on the "down low" who may utilize the Grindr application, but not hang out in traditionally gay neighborhoods and venues.

The resulting sample is biased in several ways that must be acknowledged. First, the sample is comprised only of men who own smartphones, and may thus bias the sample toward men of higher economic strata. Second, relative to the population of Los Angeles, Black/African American MSM were under-represented in this sample [34]. Third, the sample is comprised only of men who use Grindr, 
Citation: Rice E, Holloway I, Winetrobe H, Rhoades H, Barman-Adhikari A, et al. (2012) Sex Risk among Young Men who have Sex with Men who use Grindr, a Smartphone Geosocial Networking Application. J AIDS Clinic Res S4:005. doi:10.4172/2155-6113.S4-005

Page 7 of 8

which may preclude many men in monogamous relationships or who are afraid of having such an application on their phone because it might be seen by others. A focus group of Los Angeles YMSM shared that a common practice among YMSM is to remove the application from their phones when entering into a monogamous relationship as a sign of commitment and fidelity to that partner (USC focus group, June 2011). Fourth, the sample includes only men who spent time in close proximity to the recruiter; therefore, men who never worked, lived, or socialized in the neighborhoods in which we recruited were excluded. This latter limitation may partly explain the relatively low number of African American participants, given that other studies have encountered difficulty recruiting African American participants in West Hollywood [35].

Beyond these limitations associated with the sampling strategy, there are other limitations to this study. The data collection relies on self-reports, which may not accurately represent sexual risk-taking behavior; however, the anonymity of the questionnaire aimed to reduce social desirability bias. Finally, these data were cross-sectional and so only associations (not causality) can be asserted in these findings.

Despite these limitations, we believe that these data provide compelling evidence for future directions in study methodology and research with YMSM, specifically related to geosocial networking applications. The geo-locating aspects of the application made it easy for two part-time research assistants to recruit a sample of 195 YMSM in only 56 days. Our procedures were relatively simple, making it possible to achieve a true random sample, something that is often difficult within community-based research. Moreover, these data suggest a new frontier in HIV prevention research. As YMSM are becoming active consumers of geosocial networking applications, it would behoove the field to conduct more research on how YMSM are using these applications, if there are differences in behaviors based on various applications, and how YMSM compare to heterosexual young adults who are engaging in similar geosocial networking partner-seeking behaviors.

Given the many non-sexual uses of Grindr, geosocial networking applications may present opportunities in community building for YMSM, presenting a potential new area for intervention. However, as these applications are used for partner seeking, it seems critical to address how such applications can incorporate HIV prevention content. The Grindr website (grindr.com) features its own health page providing accurate information from the Centers for Disease Control and Prevention and the Pride Institute regarding sex and substance use risk behaviors, along with links to other credible resources and one to locate nearby HIV testing services [36], though this information is not contained within the Grindr application. As such, geo-locations of HIV testing or condom distribution sites and HIV testing information may be incorporated into these geosocial networking applications, so as to further encourage safer sex and HIV testing among its users.

\section{References}

1. Centers for Disease Control and Prevention (2011) HIV among Youth: National Center for HIVIAIDS, Viral Hepatitis, STD, and TB Prevention, Division of HIV AIDS Prevention.

2. Al-Tayyib AA, McFarlane M, Kachur R, Rietmeijer CA (2009) Finding sex partners on the internet: what is the risk for sexually transmitted infections? Sex Transm Infect 85: 216-220.

3. Bull SS, McFarlane M, Rietmeijer C (2001) HIV and sexually transmitted infection risk behaviors among men seeking sex with men on-line. Am J Public Health 91: 988-989.

4. Garofalo R, Herrick A, Mustanski BS, Donenberg GR (2007) Tip of the Iceberg: young men who have sex with men, the Internet, and HIV risk. Am J Public Health 97: 1113-1117.

5. Liau A, Millett G, Marks G (2006) Meta-analytic examination of online sexseeking and sexual risk behavior among men who have sex with men. Sex Transm Dis. 33: $576-584$

6. Mustanski B, Lyons T, Garcia SC (2011) Internet use and sexual health of young men who have sex with men: a mixed-methods study. Arch Sex Behav 40: $289-300$.

7. Mustanski BS (2007) Are sexual partners met online associated with HIV/ST risk behaviours? Retrospective and daily diary data in conflict. AIDS Care 19: 822-827.

8. Lloyd D (2010) Grindr iPhone App: GPS for the Sex Superhighway. The Huffington Post: Politics Daily.

9. Yiannopoulos M (2010) Grindr: combatting loneliness or cruising ground for gays?. The Telegraph.

10. Grindr (2010) Grindr Users Around the World.

11. Grindr (2011) Grindr Surpasses Three Million Users in 192 Countries. prnewswire.com: PR Newswire.

12. Chiasson MA, Hirshfield S, Remien RH, Humberstone M, Wong T, et al. (2007) A comparison of on-line and off-line sexual risk in men who have sex with men: an event-based on-line survey. J Acquir Immune Defic Syndr 44: 235-243.

13. Jenness SM, Neaigus A, Hagan H, Wendel T, Gelpi-Acosta C, et al. (2010) Reconsidering the internet as an HIVISTD risk for men who have sex with men. AIDS Behav 14: 1353-1361.

14. Kim AA, Kent C, McFarland W, Klausner JD (2001) Cruising on the Internet highway. J Acquir Immune Defic Syndr 28: 89-93.

15. Horvath KJ, Rosser BR, Remafedi G (2008) Sexual risk taking among young internet-using men who have sex with men. Am J Public Health 98: 1059-1067.

16. Rosser BR, Oakes JM, Horvath KJ, Konstan JA, Danilenko GP, et al. (2009) HIV sexual risk behavior by men who use the Internet to seek sex with men: results of the Men's INTernet Sex Study-II (MINTS-II). AIDS Behav 13: 488-498.

17. Bauermeister JA (2012) Romantic ideation, partner-seeking, and HIV risk among young gay and bisexual men. Arch Sex Behav 41: 431-440.

18. Bolding G, Davis M, Hart G, Sherr L, Elford J (2007) Where young MSM meet their first sexual partner: the role of the Internet. AIDS Behav 11: 522-526.

19. McFarlane M, Bull SS, Rietmeijer CA (2002) Young adults on the Internet: risk behaviors for sexually transmitted diseases and HIV(1). J Adolesc Health 31: 11-16.

20. Kubicek K, Carpineto J, McDavitt B, Weiss G, Kipke MD (2011) Use and perceptions of the internet for sexual information and partners: a study of young men who have sex with men. Arch Sex Behav 40: 803-816.

21. Brown G, Maycock B, Burns S (2005) Your picture is your bait: use and meaning of cyberspace among gay men. J Sex Res 42: 63-73.

22. Davis M, Hart G, Bolding G, Sherr L, Elford J (2006) E-dating, identity and HIV prevention: theorising sexualities, risk and network society. Sociol Health IIIn 28: $457-478$.

23. Davis M, Hart G, Bolding G, Sherr L, Elford J (2006) Sex and the Internet: gay men, risk reduction and serostatus. Cult Health Sex 8: 161-174.

24. Vernon P (2010) Grindr: a new sexual revolution? The Guardian.

25. Lee E, Mao L, McKenzie T, Batrouney C, West M, et al. (2012) Gay community periodic survey: Melbourne 2012. Sydney: National Centre in HIV Social Research, The Univeristy of New South Wales.

26. Jenkins RA (2011) Recruiting substance-using men who have sex with men into HIV prevention research: current status and future directions. AIDS Behav.

27. Ford WL, Weiss G, Kipke MD, Ritt-Olson A, Iverson E, et al. (2009) The Healthy Young Men's Study: sampling methods to recruit a random cohort of young men who have sex with men. J Gay Lesbian Soc Serv 21: 357-373.

28. Harawa NT, Greenland S, Bingham TA, Johnson DF, Cochran SD, et al. (2004) Associations of race/ethnicity with HIV prevalence and HIV-related behaviors among young men who have sex with men in 7 urban centers in the United States. J Acquir Immune Defic Syndr 35: 526-536. 
Citation: Rice E, Holloway I, Winetrobe H, Rhoades H, Barman-Adhikari A, et al. (2012) Sex Risk among Young Men who have Sex with Men who use Grindr, a Smartphone Geosocial Networking Application. J AIDS Clinic Res S4:005. doi:10.4172/2155-6113.S4-005

29. Remafedi G (2002) Suicidality in a venue-based sample of young men who have sex with men. J Adolesc Health 31: 305-310.

30. Stueve A, O'Donnell LN, Duran R, San Doval A, Blome J (2001) Time-space sampling in minority communities: results with young Latino men who have sex with men. Am J Public Health 91: 922-926.

31. Sumartojo E, Lyle C, Choi K, Clark L, Collins C, et al. (2008) Prevalence and correlates of HIV testing in a multi-site sample of young men who have sex with men. AIDS Care 20: 1-14.

32. Horvath KJ, Rosser BR, Remafedi G (2008) Sexual risk taking among young internet-using men who have sex with men. Am J Public Health 98:10591067.
33. Hospers HJ, Kok G, Harterink P, de Zwart O (2005) A new meeting place: chatting on the Internet, e-datng and sexual risk behaviour among Dutch men who have sex with men. AIDS 19: 1097-1101.

34. Nakelsky S, Hu V, Kahn E, Sheng Z, Taylor L, et al. (2011) Using HIV incidence surveillance data to examine racial/ethnic disparities in HIV testing behaviors among YMSM in Los Angeles County.

35. Holloway IW, Cederbaum J, Ajayi A, Shoptaw S (In press) Where are the young men in HIV prevention research? Young men who have sex with men in Los Angeles County comment on HIV prevention programs and research. J Prim Prev.

36. Grindr (2010) Grindr Health. 